\title{
Engineering Judgment and Natural Circulation Calculations
}

\author{
J. C. Ferreri ${ }^{1,2}$ \\ ${ }^{1}$ Autoridad Regulatoria Nuclear, Avenida del Libertador 8250, 1429 Buenos Aires, Argentina \\ ${ }^{2}$ CONICET and National Academy of Sciences of Buenos Aires, Argentina
}

Correspondence should be addressed to J. C. Ferreri, jferreri@arn.gob.ar

Received 9 September 2010; Accepted 29 November 2010

Academic Editor: Alejandro Clausse

Copyright ( 2011 J. C. Ferreri. This is an open access article distributed under the Creative Commons Attribution License, which permits unrestricted use, distribution, and reproduction in any medium, provided the original work is properly cited.

\begin{abstract}
The analysis performed to establish the validity of computer code results in the particular field of natural circulation flow stability calculations is presented in the light of usual engineering practice. The effects of discretization and closure correlations are discussed and some hints to avoid undesired mistakes in the evaluations performed are given. Additionally, the results are presented for an experiment relevant to the way in which a (small) number of skilled, nuclear safety analysts and researchers react when facing the solution of a natural circulation problem. These results may be also framed in the concept of Engineering Judgment and are potentially useful for Knowledge Management activities.
\end{abstract}

"No man outside his specialty is not credulous..." Jorge Luis Borges, "The secret miracle", Fictions

\section{Introduction}

The concept Engineering Judgment (EJ) is sometimes invoked to support the validity of technical assertions based on the subjective judgment of experts. This is particularly true when uncertainty prevails regarding the data at hand, in opposition to statistically valid data sets. Many relevant technical decisions are based on this type of EJ. In particular, the assignment of subjective probabilities to rarely occurring events is a usual example of this particular use of EJ. The statement "educated guessing" used to be an alternative nomenclature to denominate this somewhat arbitrary, nonscientific, way of value assignment to parameters. The nuclear corporation is sensitive to these aspects and one of the general conclusions of a nuclear safety specialists meeting, see Aksan [1], was to "Minimize need for expert judgment as far as practicable". Needless to say, this is also the more common cause for public and nongovernmental organizations complaints regarding risk and cost-benefit analyses of installations. Public and NGOs opposition to chemical, nuclear and many other types of industrial emplacements are, quite frequently, the consequence of their negative perception of said risk-benefit studies.
EJ is really at the base of the usual way of engineering data analysis. It is the case of deciding whether or not a calculated set of results can be considered a valid one. In this paper, applications of EJ deal with the computer prediction of the stability of natural circulation (NC) flows (jargon for natural thermal convective flows) in hydraulic loops of interest in the nuclear industry.

The simplest approximation will be considered, namely, one-dimensional (1D), almost incompressible flow in single phase. It may be argued that it is a rather simplistic problem, because real life installations show much more complicated situations. However, most of the calculations performed under these restrictive hypotheses pose some challenges that must be solved on the basis of EJ if this is understood, as mentioned above, as the process performed to determine the validity of a given set of computer results.

The emphasis of this paper is not on the two basic steps of computer code development, namely, verification and validation. These steps are assumed as already done. Here, the verified and validated codes are used to analyze the behavior of quite simple loops, either theoretical or experimental ones, with the main interest focused on assessing the results. As a consequence, some insights are derived to account for 
the effects of discretization and closure correlations. One aspect that will deserve particular consideration is whether to stop for the search of perfection in the achieved results, this in the light of lack of really valid experimental data allowing for partial validation or lack of exact solutions for the problem under analysis (the vast majority of real life engineering problems) with different codes.

Perhaps, before starting the analysis, it may be useful to excerpt some considerations by Scannapieco and Harlow [2] on the role of computational predictions: "In as much as we can simulate reality, we can use the computer to make predictions about what will occur in a certain set of circumstances. Finite-difference techniques can create an artificial laboratory for examining situations which would be impossible to observe otherwise, but we must always remain critical of our results. Finite-differencing can be an extremely powerful tool, but only when it is firmly set in a basis of physical meaning. In order for a finite-difference code to be successful, we must start from the beginning, dealing with simple cases and examining our logic each step of the way". Harlow was one of the most talented experts in Computational Fluid Dynamics, who leaded the famous Group T3 at Los Alamos Scientific Laboratory in the 70-80s.

From the regulatory point of view, the need for independent safety analysis cannot be sufficiently emphasized. It must be understood that the same engineering data most probably will generate different results, even using the same code and the same (agreed with the licensee) criteria for discretization. Differences would arise from choosing different code options or what is the code user interpretation of the agreed criteria. In passing, the importance of EJ may be once again exemplified by the following excerpt from the work of Shotkin [3]: "It should be stressed that the staff does not rely solely in computer analyses, but rather use the analyses as a tool to help guide understanding of plant behavior in conjunction with Engineering judgment, hand calculations, data analysis, and experience with plant operation". Also: "It must be continually emphasized that code results must always be used with cautionary Engineering judgment. This is true even for those uses where the code has been explicitly assessed against data because user choices and input deck errors may influence the calculation results".

In what follows, some examples coming from previous work by the author and his colleague at the University of Pisa, Professor Walter Ambrosini, are reviewed and presented. These results will be the support for a part of the present contribution.

Also in relation to the aforementioned work, a theoretical experiment was performed, aimed at testing how a bunch of skilled, active and young nuclear safety analysts and researchers would react when faced with solving some puzzling results of the use of a systems thermal hydraulic code and an in house developed thermal hydraulic code. The information given to these people was somewhat biased to provoke an unneeded sophistication of the analysis. The results showed that this bias was (regretfully) successful. Some other aspects on scientific information as presented in technical journals are discussed and the lessons learned are made explicit. These aspects would also be potentially useful for Knowledge Management (KM) activities.

It must be mentioned that the subjects herein discussed are some of the more important aspects of safety evaluations and this brief, quite restricted presentation may, hopefully, contribute some emphasis on them.

\section{The Search for Convergence of Results}

This is, perhaps, the easiest step in computational analysis of engineering problems but only conceptually. In fact, it means that grid size, as measured by some suitable norm, is compatible with the accuracy of resolution of some type of boundary layer. This may be a momentum boundary layer as in the vicinity of a wall, the depth of heat penetration in a solid, or the time history of some suitable dependent variable as a function of its time scale, among many other possible examples. What must be considered is that a given boundary layer behavior must be solved accurately enough. Searching for grid convergence is not a too costly activity in simple integration domains, like the 1D cases herein considered. This is not the case in multidimensional domains. In the latter, the use of multiple scale calculations tends to keep detail and accuracy at an appropriate level in the entire integration domain. Shape and size variation of computational cells affect the global accuracy.

In the case of NC in unstable flow conditions analyzed using time domain computer codes, the problem consists in using a spatial discretization fine enough as to minimize the amount of numerical diffusion. This numerical diffusion is sometimes added in the process of solution as a consequence of the inherent properties of the discrete scheme. This diffusion is usually associated with first-order spatial discretization. It may be argued that using spatial $\boldsymbol{O}(1)$ numerical schemes should not be recommended in general. However, most engineering thermal-hydraulic systems codes use this approximation to circumvent a worse limitation: the ill-posedness of governing equations.

The interaction of flow stabilization and discretization may be exemplified resorting to results cited in Ferreri and Ambrosini [4], as shown in Figure 1, where the flow rate in a simple loop of Figure 2 was obtained using a finitedifference scheme $\boldsymbol{O}(\Delta x, \Delta t)$ known as forward time (Euler) upstream space (FTUS in short), 1000 spatial nodes, and a cell Courant number $(\equiv U \cdot \Delta t / \Delta x), C=0.8$. The results are compared with the ones obtained using a modal expansion, which is free of numerical diffusion, with 500 modes and adding the numerical diffusion nearly corresponding to the previous approximation. It may be observed that the results are nearly the same. Then, it may be concluded that the usual interaction between the numerics and the physics persists in this nonlinear case.

The results by Ferreri and Ambrosini [4] showed how using different order schemes could be useful to get improved convergence of results to some limiting accuracy. Perhaps the most interesting results were showing how usual approximations of piping systems related to nuclear industry could be nonconservative from the point of view of safety. In fact, revisiting a pioneering work by Welander [5], a stability map 


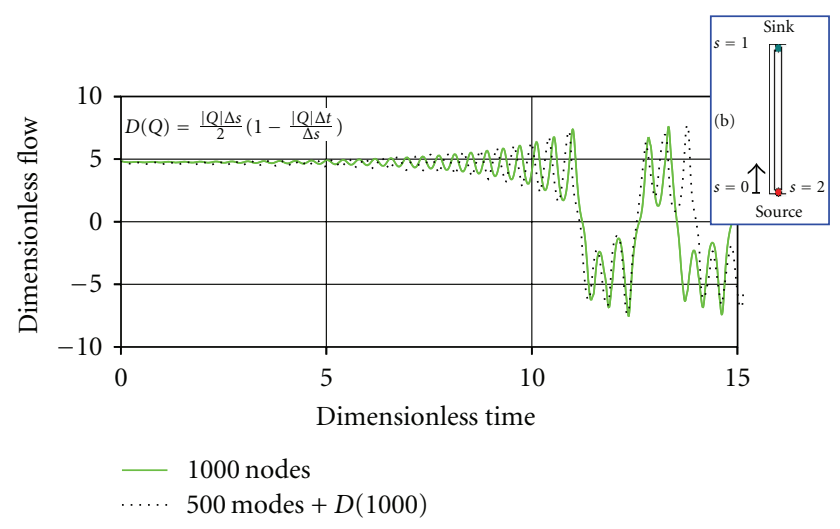

FIGURE 1: The flow rate for the FTUS scheme using 1000 nodes and its simulation using a modal expansion of 500 modes and adding the numerical viscosity corresponding to 1000 nodes.
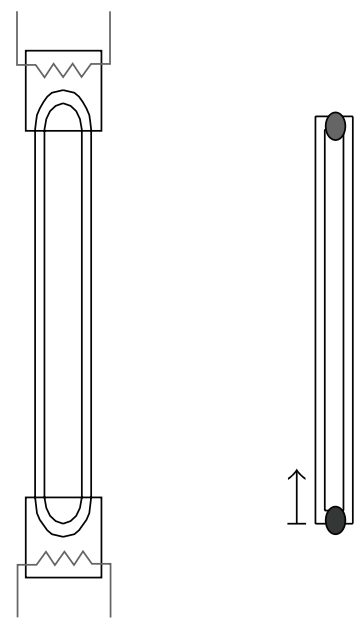

FIGURE 2: A schematic view of a natural circulation loop, adapted from Welander (1967).

was determined. It corresponds to a two pipes loop $10 \mathrm{~m}$ high and $0.1 \mathrm{~m}$ in diameter, with a concentrated heat source at the bottom and an opposite heat sink at the top, as the one shown in Figure 2.

The analytical stability map is the one in Figure 3, where a working point corresponding to an unstable flow condition was set. Then the map was constructed by calculation with the FTUS approximation and the effect of the number of nodes was determined. In the maps following, $\alpha$ and $\varepsilon$ are two nondimensional parameters that measure, respectively, the buoyancy driving force and the resisting friction force in the loop.

Figure 4 shows that, as the number of nodes increases, the unstable region in the map progressively converges to the theoretical stability boundary (SB). Then, for the point under analysis, flow changes from a stable condition to an unstable one. Then, the evaluation of this system goes from a non-conservative stability condition evaluation towards a conservative, real unstable one. Predicting the system to be stable is, obviously a noncorrect, dangerous situation in this case.

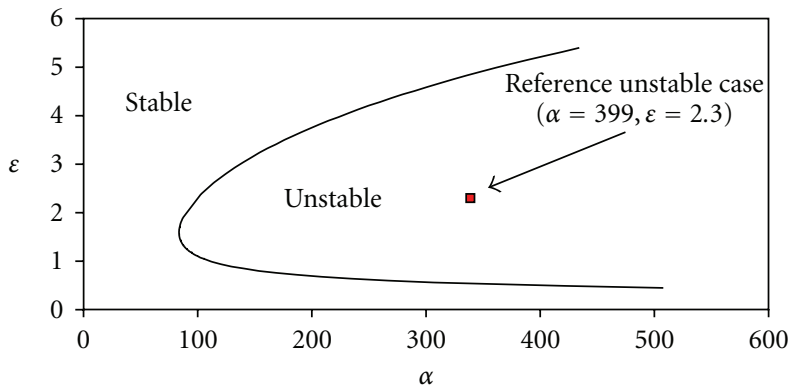

FIGURE 3: Theoretical stability map for the positive flow steady-state conditions.

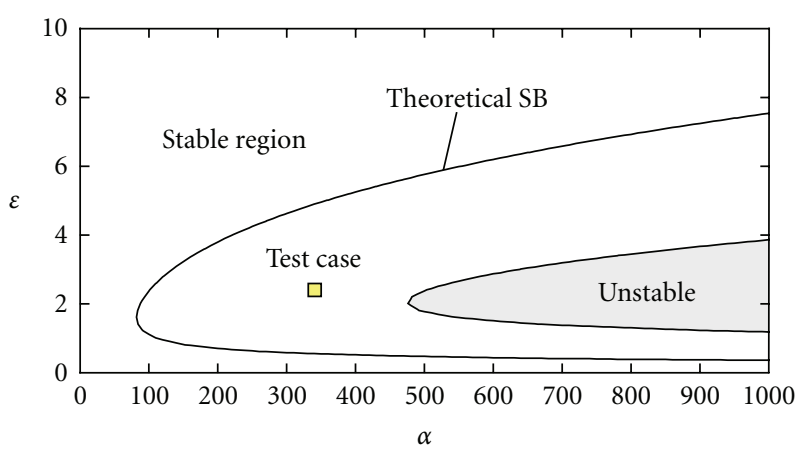

(a)

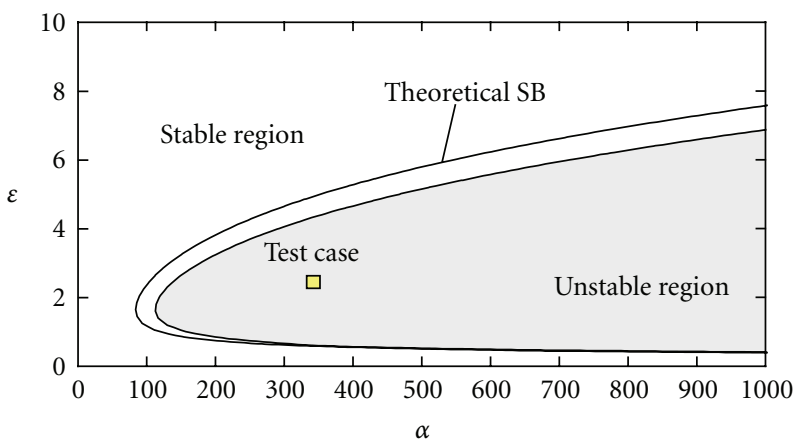

(b)

FIGURE 4: (a) Stability map with 30 nodes per leg. (b) Stability map with 100 nodes per leg.

The interesting consideration here is that discretizing a pipe $10 \mathrm{~m}$ long and $0.1 \mathrm{~m}$ internal diameter in volumes $0.3 \mathrm{~m}$ long seems natural to a systems code user, at least as a compromise between computational cost and expected system behavior. Then, assuming that the system is expected to perform in a stable way, EJ must be used to decide on various aspects, namely, (a) the system satisfies the design goals; (b) the numerical model is appropriate; (c) the computer code is applicable; (d) the discretization is adequate and does not mask some unexpected behavior; (e) results are converged. These questions are of great importance for the safety evaluation of nuclear installations. Furthermore, as they seem natural, they have also been considered in the so-called Code Scaling, Applicability and 
Uncertainty (CSAU) evaluation methodology; see reference [6], a United States Nuclear Regulatory Commission's major documented way to assess the traceability of nuclear safety analyses. Also, the need for the qualification of codes and their users arises in a natural way and this, incidentally, has also been the subject of much analysis; see for example, the discussions in [7], among others.

Another problem arises when two independent code results are compared. A general, advanced thermal-hydraulic systems code like RELAP5, see Carlson et al. [8], and another of restricted validity can be both applied to a particular physical situation for which the second is known to be applicable. In NC flows in single phase, the mass flow rate scales with the $1 /$ third power of the heat input to the system. Then, a difference of $10 \%$ in heat input leads to only $3.2 \%$ in flow rate. This last difference is small and acceptable in most situations, given the uncertainties in codes and their closure correlations, but covers a significant one in power. Deciding when it is possible to accept this difference poses some challenge for large, complex systems and requires applying EJ again. Regarding convergence of results, some care must be also taken when lumped parameter simulations are used. In Ferreri et al. [9, 10], a lumping criterion for concentrated heat source/sink was developed, which eliminates the lack of convergence due to heated length in an FTUS finite-differences scheme applied to the abovementioned problem. These results arose from applying EJ to this lack of convergence.

\section{The Effect of Closure Correlations}

Related to the previous search for convergence of results, there is another aspect to be taken into account. It is whether an accepted, commonly applied closure correlation is appropriate to describe the physics of the problem under analysis. Closure correlations serve to set a system of conservation equations closed. Most commonly, they include interface and interphase relations like friction laws, heat transfer correlations, phase slip velocity specification, and many others. In this section, the effects of using different versions of the macroscopic friction law will be discussed. It is important to say, from the very beginning, that if the results of a computer prediction are not known (the usual case in engineering calculations), then using accepted closure correlations is a basic tenet. There is nothing to be argued against this practice. On the contrary, it is supported by common sense and EJ. On the other side, it must be noted that unstable, time reversing flows always traverse a laminarturbulent flow transitional region. The time scale associated to these reversals may affect the influence of the transitional regime.

It may be interesting to consider firstly the effect of friction law in the stability map of a toroidal loop. This geometry is amenable to analytical and numerical analysis and has been the subject of research since decades ago. An example of this may be found in Ferreri and Doval [11] and Figure 5 shows, without makeup, how the system behaved changing the nodalization, showing the usual damping of the FTUS finite-differences scheme.

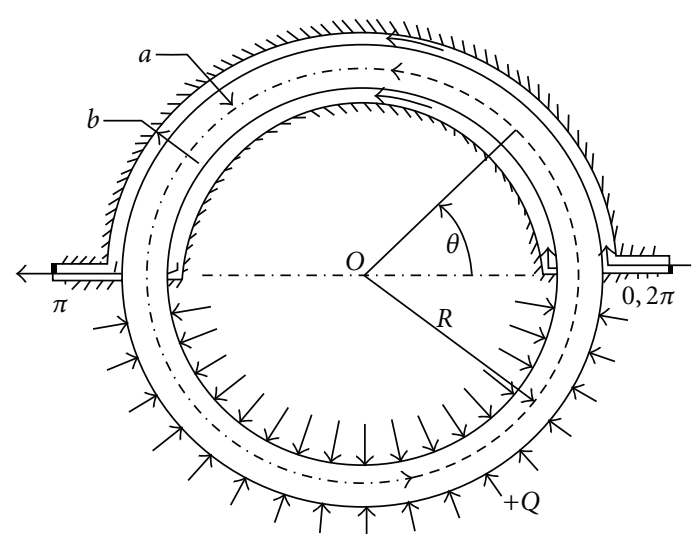

(a)

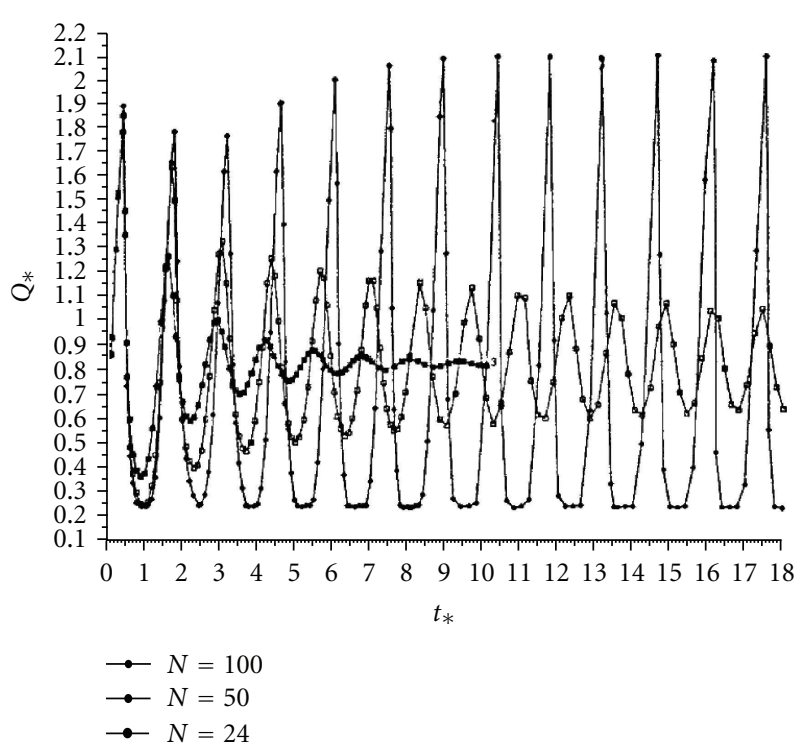

(b)

FIgURe 5: A toroidal loop with fixed input heat and dynamic heat exchanger.

Far more recently, in Ferreri and Ambrosini [4], the effects of the friction laws on the stability maps of a similar system were analyzed. Figure 6(a) shows the most usual correlations for the friction factor in a tube, as a function of the Reynolds number. The one signaled as Churchill law is an adequate fitting to the Moody's law used for smooth tubes in engineering calculations. Figure 6(b) shows how the neutral stability boundary is affected by the particular choice of the friction factor variation at the transition of the flow from laminar to turbulent. The variation is also predicted using the FTUS methodology and a modal expansion solution of the governing equations. Now, a more realistic situation will be analyzed.

Let us now consider the following experimental results, Vijayan et al. [12], dealing with NC flow in a simple square loop. The loop consists of a $23.2 \mathrm{~mm} \mathrm{I.D.} \mathrm{glass}$ pipe, having $2.1 \mathrm{~m}$ vertical legs, and equipped with $0.8 \mathrm{~m}$ long electrically heated and fluid cooled horizontal sections. 


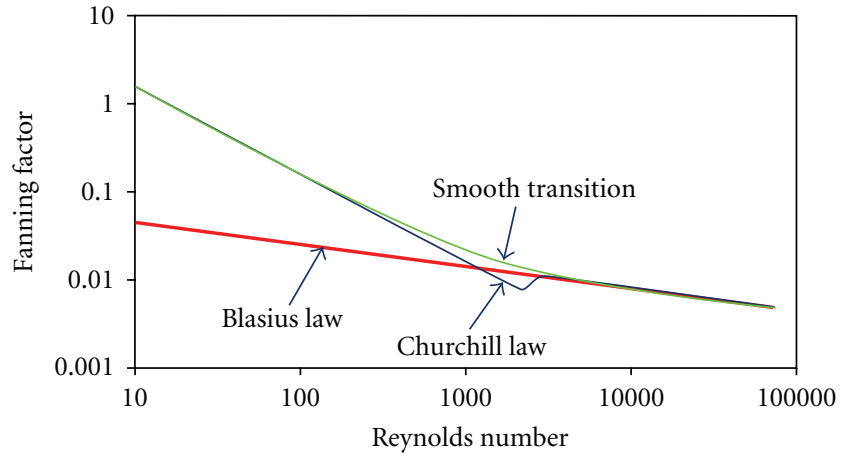

(a)

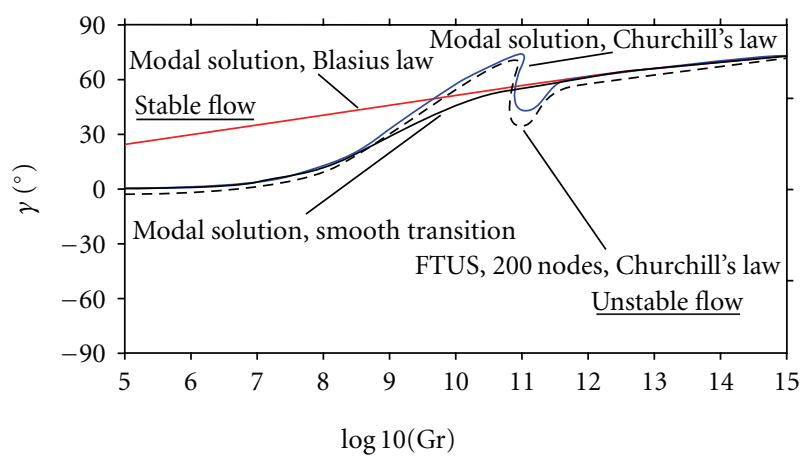

(b)

FIGURE 6: (a) The variation of friction factor with Reynolds number. (b) The stability map as a function of the friction law.

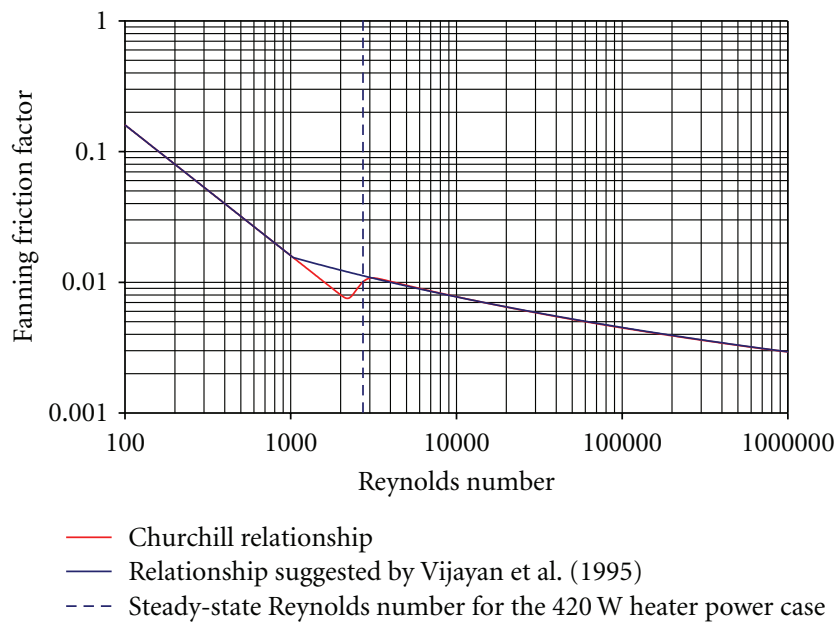

FIGURE 7: Friction factor as a function of Reynolds number.

The latter consists of a pipe-in-pipe heat exchanger, fed by relatively cold water and at prescribed flow rates. This loop showed unstable NC flow conditions for a heat power input of $420 \mathrm{~W}$. These results have been simulated by a set of two codes described in Ambrosini and Ferreri [13]. Figures 7 and 8 show the results of the predictions using Churchill's approximation. As may be observed, the map shows a band of stable flow condition. Figure 9 shows the map for the same

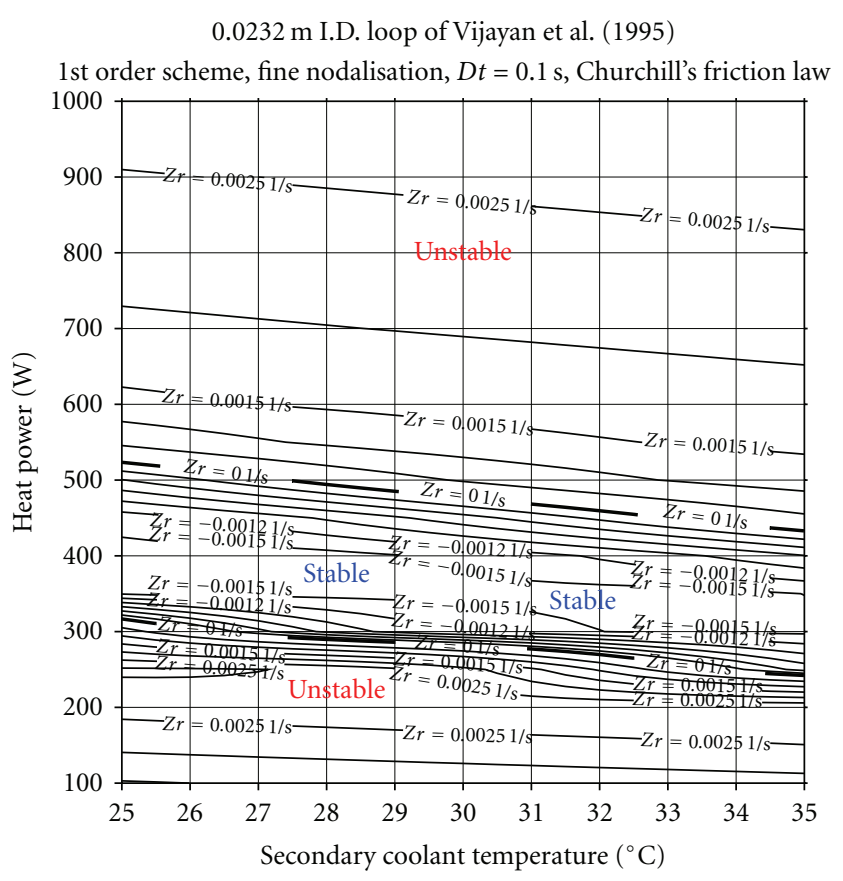

FIgURE 8: Stability map using Churchill's law.

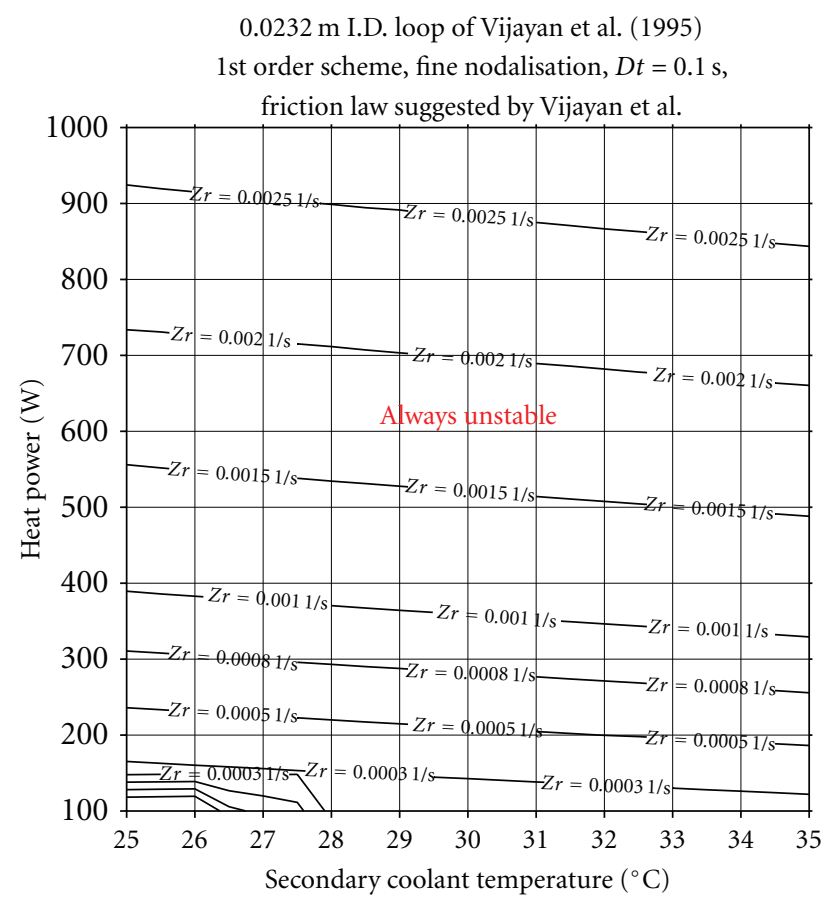

FIgURE 9: Stability map using Vijayan's suggested friction law.

conditions using the friction law as suggested in Vijayan et al. [12]. The flow is always unstable, as the experiments also indicate.

The calculation using the codes of Ambrosini and Ferreri [13] with the correlation by Vijayan et al. [12] permitted to recover a condition similar to the one in Figure 9, that is, a completely unstable map. Now, the following may be concluded: the transition laws adapted to link correlations 
for laminar and turbulent flow as adopted in thermalhydraulic codes are under question in unstable flows. It was shown that a nonmonotonous transition branch in the correlating curve may lead to predict stability, whereas experimental observations show unstable behaviour. Again the condition is not conservative.

It is somewhat difficult to establish an EJ criterion to deal with this situation. Perhaps, the conclusion in Ambrosini et al. [14] can be repeated here: the validity of the traditional claim for the inapplicability of the forced convection friction correlations in natural circulation conditions appears to be rather dependent on the geometry of the loop. In fact, though in some literature works including comprehensive reviews, recommendations are given to use friction laws providing larger friction factors than in forced flow, the work of Vijayan et al. [12] seems to suggest that classical laminar and turbulent friction correlations perform reasonably well in rectangular loops. It is so when appropriate localised pressure drop coefficients are included in the models to account for the effect of bends and other discontinuities. Nevertheless, what is clear is that transitional flows must be evaluated quite carefully, testing the effects even of the most classical closure correlations.

\section{Testing the Possibility of Continued Knowledge Development in NC}

It may be accepted, loosely paraphrasing Kuhn, that in the evolution of science there are sudden jumps in knowledge, followed by stability periods of consolidation and accumulation of related information. The last century shows several examples when, after the foundations of a new theory are well established in a particular field, an explosive increase in the number of related scientific publications occurs which, paradoxically, is the true symptom of stability. This situation persists until new evidence cannot be explained in terms of the prevailing paradigm. Typically this leads to the formulation of a new paradigm and the cycle restarts.

In addition, well-known concepts may experience a revival after some years of lethargy. This applies in the case of learned journals too. There are several factors contributing to the last mentioned situation but it is the author's opinion that the contribution from reviewers is not the least. It is obvious that as time elapses, the list of peers change and the newer ones may not have enough time (or predisposition) to read previous, "old" literature. In this way they may be unwittingly prone to recycle information. Researchers that have been publishing their findings since thirty years ago may be conscious witnesses of this phenomenon.

The reading of an essay on automata by Garassa [15] suggested what will be proposed in the following, with the aim of showing the possibility of pushing the order in a period of stability to its limits through almost automatic knowledge advancement.

The general proposal was remarkably simple:

The Almost Automatic Exploration of Knowledge Niches to Get Additional, Supporting, Continuing Contributions. In order to test the feasibility of this approach, a theoretical experiment was devised. The experiment has been carried out with the contribution of several young, experienced, professionals belonging to several groups with theoretical and experimental skills in nuclear engineering. They are professionally active in the field and were willing to participate in a "theoretical experiment in KM". The participants had previous working background or recent training on the addressed subject. Only e-mail contact was used. The interest of this approach may be, hopefully, evident in what follows.

To accomplish this goal, the relevant issue was performing a theoretical experiment to test the possibility of continued, "normal" development of knowledge by juniors in a selected niche of knowledge, without interacting with seniors.

4.1. Subject of Application. Arguably, the knowledge niche selected was the computation of NC flows in thermalhydraulic loops.

This has been the subject of intensive research for more than thirty years. Again, a list of publications up to 2002 may be found in Ferreri and Ambrosini [4]. On the other side, even earlier, this author also tried to put in rational terms the usual thinking in setting up computational fluid dynamics (CFD) models in a rather elementary prototype of expert system, as in Ferreri and Grandi [17]. This background leads to the present election. It should be pointed out that the long range goal of this work was incorporating the way of analysis to be described in what follows in some inference machine embedded in an expert system. Automatic inference is not new, see for example, King et al. [18] and Schmidt and Lipson [19], and would allow obtaining the minor advances like the one reported here, leaving time for more relevant research tasks. On the other side, detailed procedures for documentation and data reproducibility, see Schwab et al. [20], may also be used with advantage for this long-term goal.

4.2. Selected Bibliographical Material. The information provided consisted in full text versions of references by Ferreri and Ambrosini [4], Ambrosini and Ferreri [13], and Pilkhwal et al. [16]. Really, to use information publicly widely available online at the moment of the experiment (2007), the title, keywords, and abstract should only be used. The corresponding material of the papers cited in these references in which this author participated could also be used, but it was not suggested to the participants. The underlying idea was applying the scheme to be described below to infer the lines of research that lead to some new, unpublished data. There were two, almost evident, possible lines to be inferred, (a) the continuation of detailed studies, based on CFD codes and (b) a second one, explaining how to overcome the limitations of one-dimensional (1D) codes in the case of interest. The second, less evident, was the key leading to the set of unpublished, new results. Merging of the two techniques in a multiscale, multidomain system code was another possible solution. 
4.3. Procedure of Experimentation. The procedure followed consisted in sending a letter of invitation to the potential participants, after asking for authorization to their advisors.

The group of people included some usually working with CFD codes and some others working with so-called thermal-hydraulic system codes. The latter are basically the ones usually used to perform safety analysis of nuclear power installations as well as to get experience on their behavior through the simulation of controlled integral test facilities experiments.

The invitation letter expressed that the participant may be "aware that the management of knowledge implies taking care of heritage. Many institutions are presently suffering the effects of a long lasting lethargy. This is particularly true, although not exclusively, in the nuclear field, where seniors are beginning to retire and there is a lack of skilled, intermediate aged professionals, able to continue the activities."

It continued stating, "There is a set of results, still unpublished, which is a "natural" continuation of the line of research indicated as background material. What is expected from your participation is to infer what the aforementioned unpublished results are and the way they have been obtained, on the basis of the reading of the background material at two levels of detail as specified below. This expected outcome, of only half a page in length would imply that, what seems 'natural' to me might be easily unveiled from reading the papers." (In reality, what happened was that the author obtained the results in this way and this fact gave the opportunity to test the procedure now reported, simply by rejecting the possibility of publication of the new development. The Appendix illustrates the reasoning behind this approach.)

Then the selected material was cited, as specified before. Regarding the procedure to follow, two levels could be employed. Both started with a common premise:

(a) "Do not consult or discuss your conclusions with your advisor (I asked him/her for permission) or colleagues of your work group."

Then, the following two approaches could be followed:

(a) "Read the papers in sequence using only the title, the keywords and the abstract

(b) Draw conclusions, advancing your guess of the outcome.

(c) Write your conclusions and send them to me by e-m."

Or, in case it was felt necessary to have more detailed information, the procedure to follow could be:

(a) "Add the reading of the full text

(b) As before

(c) As before."

The selection of the references was purposely biased, the first two leading the participants to realize the limitations of one-dimensional codes. The third one stressed even more on these limitations. Also, the latter paper explicitly stated in its abstract that the difficulties could not be overcome by using $1 \mathrm{D}$ codes and that CFD codes were the natural option to follow, something that was also suggested in the second reference. The conclusion on the ultimate limitation is true as stated but, as is usually accepted in the Engineering practice when the flow pattern may be inferred from experience, a suitable nodalization can be set up, to take into account the (somewhat) complicated flow-pattern.

Then the following was suggested to the experiment participants.

As stated by Ferreri and Ambrosini [4] "Sometimes, scaling leads to the adoption of the 1D approximation; this may, in turn, hide important aspects of the system physics. A simple example of this situation consists in keeping the height of the system unchanged to get the same buoyancy; then, if the system is scaled accordingly to the power/volume ratio, the cross section area of the volume will be reduced; this leads to a much smaller pipe diameter that makes the $1 D$ representation reasonable, at the cost of eliminating the possibility of fluid internal recirculation. A workaround for this situation is providing paths for recirculation, in the form of additional, interconnected components; however, this solution may impose the flow pattern in the system and the balance between these aspects is a challenge to any practitioner in natural circulation modeling".

Also, in Pilkhwal et al. [16], it was explicitly stated "Strategies for improving the predictions of the RELAP5 code are under study by the present Authors, trying to provide the simulation of the heater in the HHHC (horizontal heater/horizontal cooler) configuration with some allowance for predicting thermal stratification phenomena".

The above-mentioned "suitable" nodalization usually comes from the application of EJ based on the simulation of experiments in similar situations. This option needs some more intuition but leads to results that may reflect the experimental trends. It also has, at least, two advantages: (a) computer time is quite small, in the order of minutes using a standard PC, as opposed to many hours using a CFD code and, (b) experience is gained, suitable for its application in reasonable extrapolations (This is the type of knowledge that may be incorporated into the system of rules in some expert system.). See also the discussion in [6].

4.4. Results. In total, more than twenty invitations were sent, distributed in five institutions at different countries. Only ten answers have been obtained, of varying degree of detail. Six answers were based on the first indication of reading and the other four on varying degrees of reading of the papers. The low number of answers may be, perhaps, attributed to the simple fact that many people think that paying attention to this type of experiment is simply not worth doing.

All the answers were conceptually correct, did not went too deep into justification, and suggested that the additional results were CFD analyses or different extensions of the third paper. What is interesting is that most of the participants are familiar and presently working with such techniques. Perhaps, these young researchers were somewhat dogmatic in considering what was written in the supplied literature and not prone to consider alternatives to what is shown in it or, perhaps worse, alternatives to their usual thinking. Another possibility is that no one was too interested in reading in detail long introductions, discussions, or conclusions. 
However, it must be emphasized that the invited people usually perform code validation to continue research and nuclear safety evaluations of advanced reactors design. Then, this may put a warning on people at the Academia, with regard to promoting appropriate use of computer resources and emphasizing on EJ, because code users may be prone to consider the least information that may lead to confirm their presumption on expected results. As a consequence, full exploitation of present computer models and codes must be emphasized at research and development groups. This may lead to saving time and resources.

Just one answer was what the author expected, suggesting among other things, the way to obtain results in the way described as (b) above, explaining how to overcome the limitations of $1 \mathrm{D}$ codes in the case of interest. This answer explicitly stated "A tentative to reproduce such behavior (stratification in horizontal pipe) by the 1D system code could be done by suitable nodalization technique (e.g., dividing the horizontal tube into two parallel parts). However special care should be given to avoid the introduction of phenomena not part of the experiment or not physical". One tenth is satisfactory as a result. Obviously, it cannot be asserted that increasing the number of participants would imply keeping a similar result.

From this experiment, it seems that the first approach to the literature analysis is not useful to continue research or, at least to explore useful alternatives to the summarized results. It is also a warning to any author (the present one is not exception) on how to write an abstract. It also seems that reasonable suggestions of further research may be obtained following the procedure quoted as the full text approach to literature analysis. Then, a more exhaustive experiment may be designed and tested based on this.

From the limited number of answers, it was concluded that

(a) the procedure, as presented, seemed reasonable. It should be tested in another field, preferably in someone dealing with a different niche of knowledge, to further test its feasibility;

(b) information, as available for browsing in presently commercially copyrighted literature is not enough to advance the knowledge, because it depends on the information that authors consider relevant to abstract.

It is suggested to continue with this type of experiment to analyze the idea proposed in this work with a wider universe of participants. The research area may deal with a different topic.

\section{Conclusions}

This paper dealt with some particular applications of Engineering Judgment to evaluate the results of computer codes application to unstable, one-dimensional, NC flows in single phase. Despite the simplicity of the systems analyzed, some problems have been exemplified that pose a challenge to the common reasoning. Perhaps, the only way to circumvent the questions of convergence of results and the effects of closure

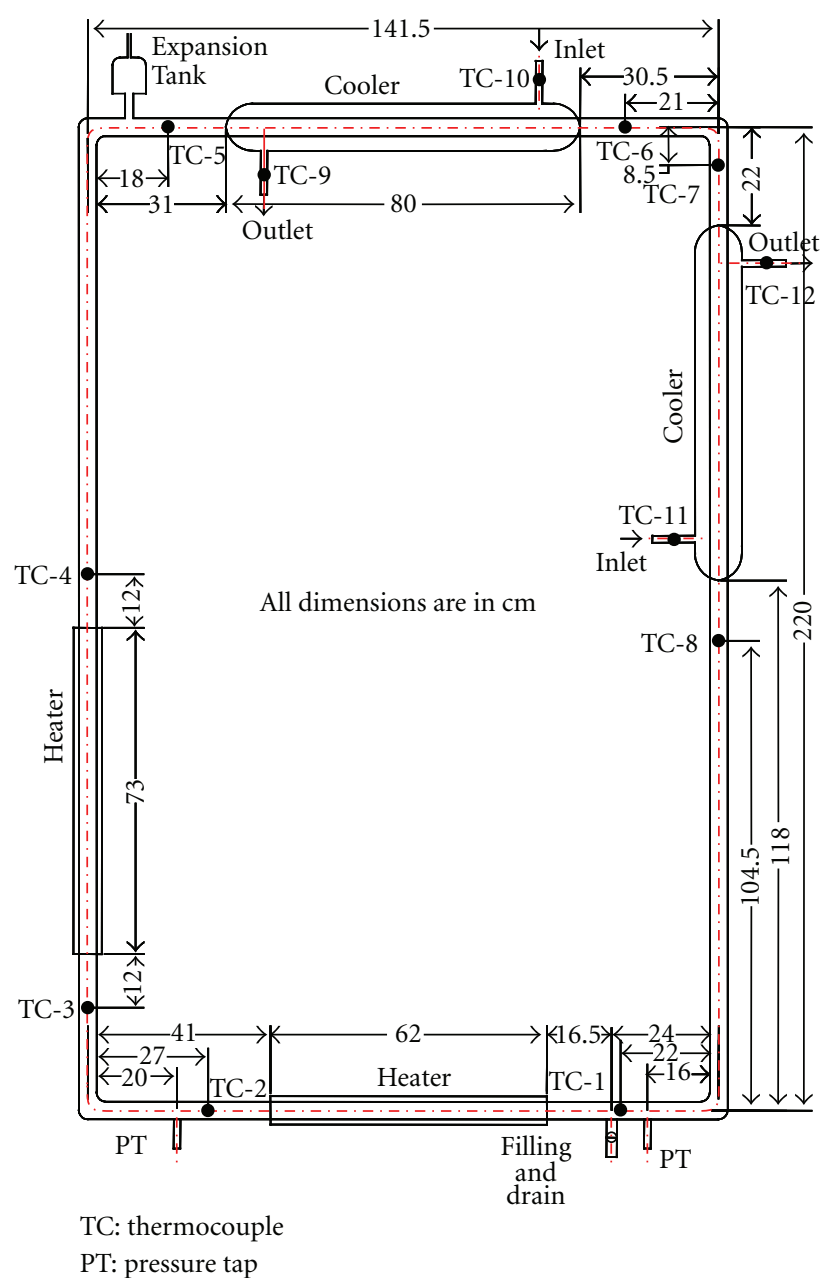

Figure 10: Sketch of the natural circulation loop [16].

correlations is to resort to sensitivity to parameters analysis. If a concluding assertion is needed, it may be that $\mathrm{EJ}$ and nondogmatism go together and that accepting clichés as working rules must be avoided. In the author's opinion, the few examples considered fully support the previous assertion.

On the other side, in order to show that it is possible to advance almost automatically in the full exploration of a knowledge niche, a number-limited, controlled experiment was performed. In so doing, the conceptual approach on the possibility of continued development by young researchers, without interaction with seniors was tested. The experiment permitted to verify that the test procedure is reasonably well founded and that the literature published so far was consistent in pointing findings and new ways amenable to exploration. The experiment may be useful in KM activities.

\section{Appendix}

As said before, a list of publications up to 2002 may be found in Ferreri and Ambrosini [4]. The results have been always related to quantify the effects of closure correlations and numerical approximations, as implemented in nuclear safety analysis codes, on the results. The particular aspect under 


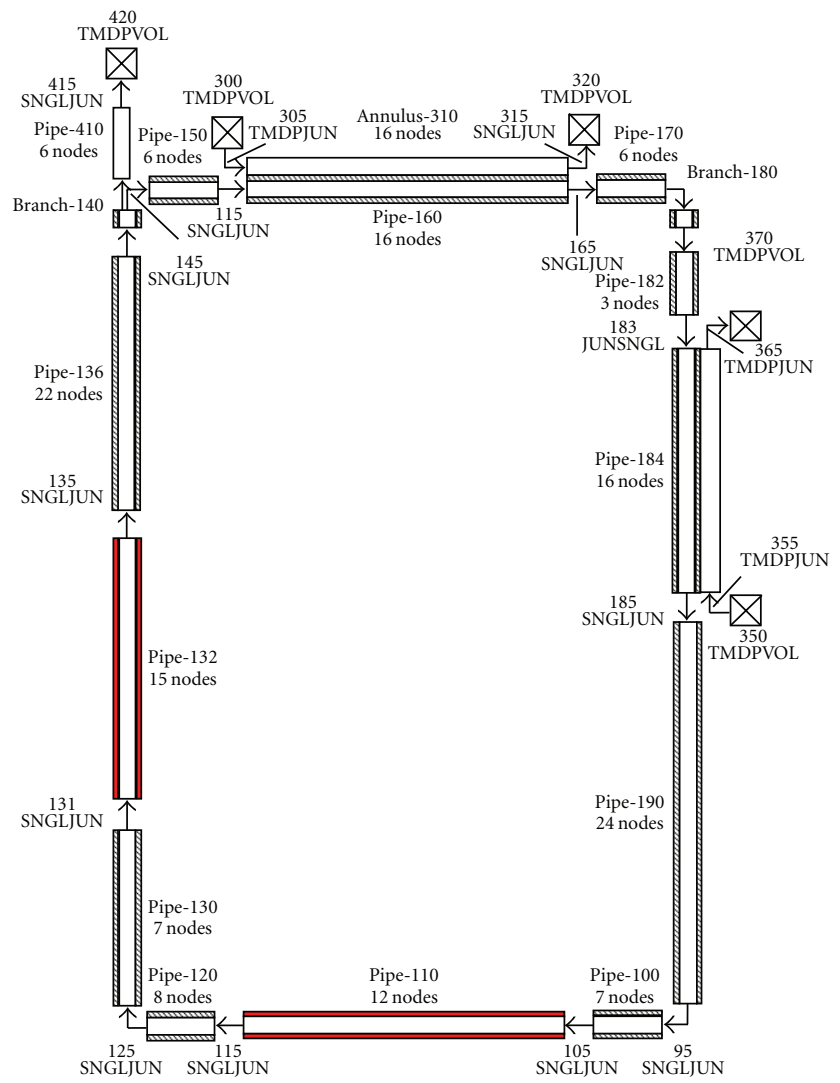

FIgURe 11: Nodalization of the experimental facility adopted for RELAP5/Mod 3.3 [16].

analysis was the unstable behavior of natural circulation flows.

The results that substantiated the experiment will be clarified in what follows, for the sake of completeness. These results partly come from Pilkhwal et al. [16]. Figure 10 shows the experimental rig that originated the results. Said rig was represented using RELAP5 and an in-house developed code named TRANLOOP. RELAP5, as developed by USNRC, see Carlson et al. [8], is one of the most widely used thermal-hydraulic systems code to perform nuclear safety revaluations. Figure 11 shows the nodalization adopted to consider the HHHC configuration mentioned above and a nominal heating power of $100 \mathrm{~W}$. The flow rate time variation in the loop is shown in Figure 12. It is a composition showing the results as obtained from (a) the experiment, (b) from RELAP5 and TRANLOOP, and (c) from a CFD code, namely, FLUENT 6.2, see Fluent Inc. [21]. As may be observed, the CFD approximation represents well the growing and persistence of the physical flow rate instabilities. This fact leads to the obvious conclusion that representing fluid stratification like a CFD code and a condition not reachable using $1 \mathrm{D}$ codes like RELAP5 and TRANLOOP may allow obtaining an adequate flow pattern description. In the case of the results obtained using RELAP5, the flow remains stagnant until the fluid starts to boil. Minute differences in temperature destabilize the flow and a cycle (like the ones visible in Figure 14 later) starts. This does not happen when

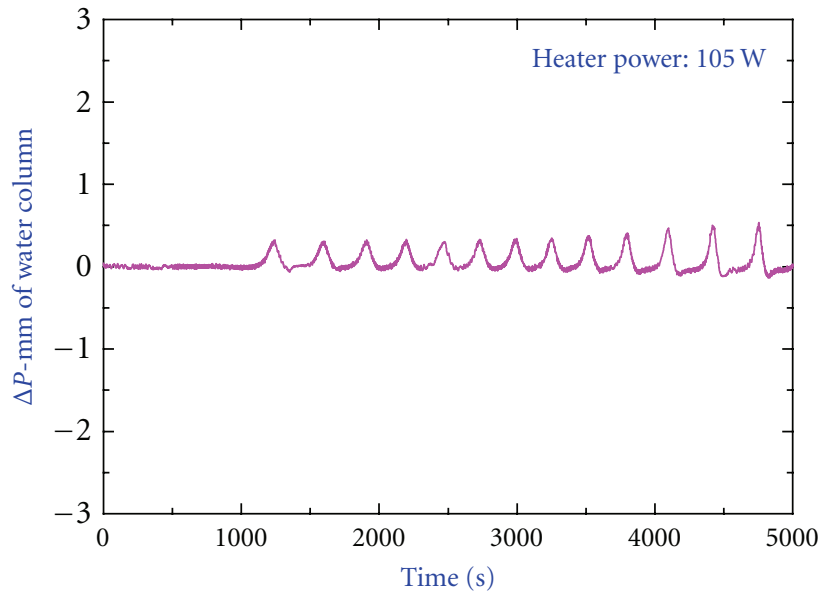

(a)

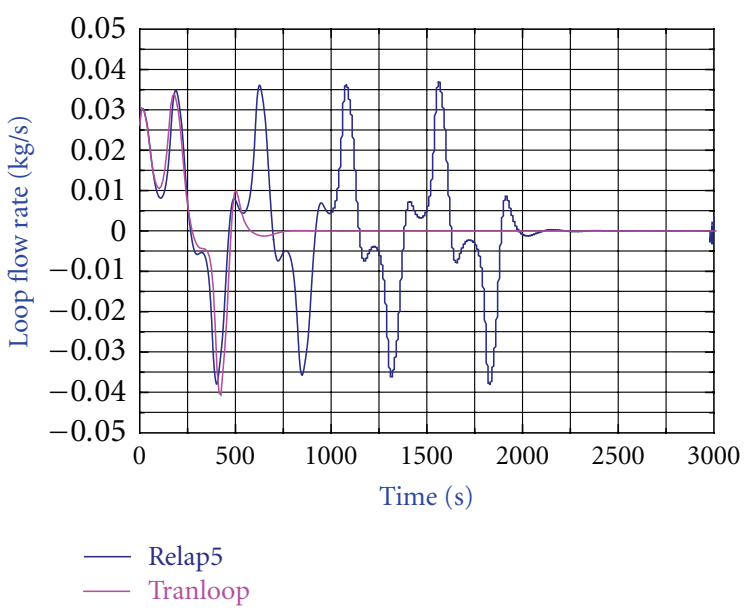

(b)

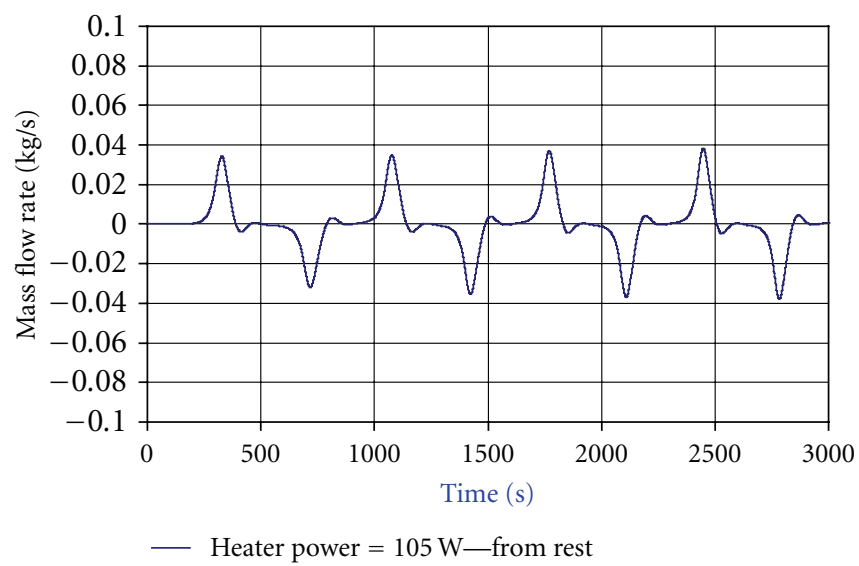

(c)

Figure 12: A composition of some results from [16]. (a) Observed unstable pressure drop behaviour for the HHHC configuration starting from rest conditions. (b) Transient behaviour predicted by TRANLOOP and RELAP5. (c) Transient behaviour predicted by the CFD model starting from rest conditions. 


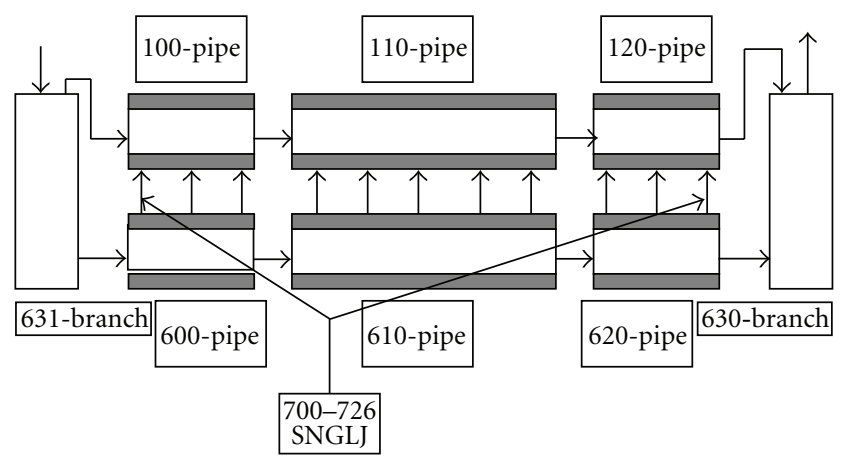

FIGURE 13: An alternative nodalization for the horizontal heater.

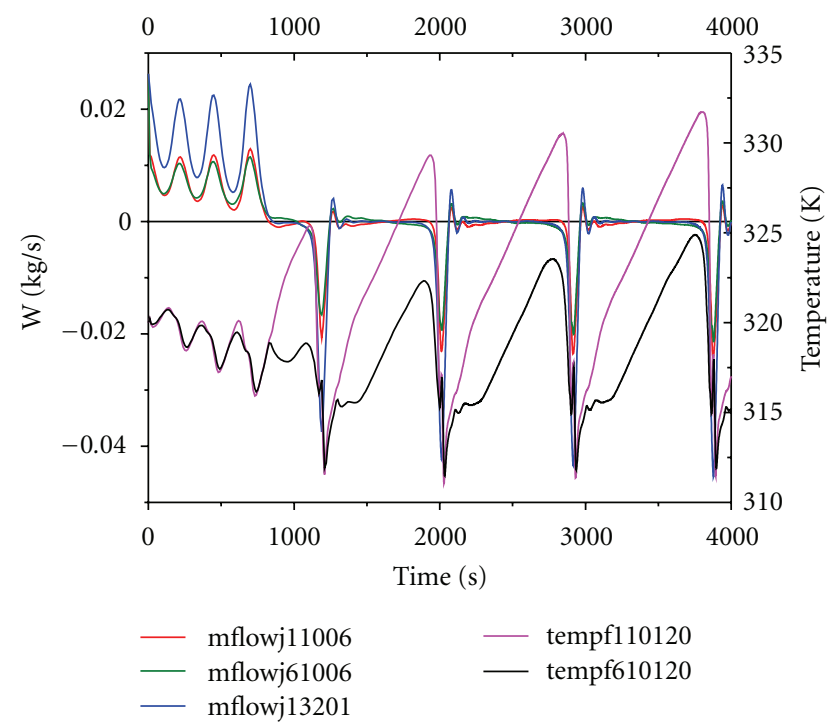

FIGURE 14: Time variation of mass flowrate and temperature using the modified nodalization.

using TRANLOOP because the Boussinesq approximation fails to reflect the physics.

As previously mentioned, some alternative nodalization using 1D codes may be considered. The one shown in Figure 13 may be one of the possible solutions in this particular case. In fact, including two interconnected parallel channels with equivalent friction and heat transfer should constitute an approximation capable to represent the expected behavior of the physical installation. Then, this nodalization was implemented using RELAP5 and the results, exemplified in Figure 14, showed that the flow instabilities may be recovered, even for lower heating rates. This nodalization of the horizontal heater did not affect the stability in the other configurations discussed in Pilkhwal et al. [16]. The different behavior is due to the transverse flows between the horizontal channels. As may be observed in Figure 14, the expected thermal stratification is roughly represented. Arguably, more parallel channels would approximate better the physical situation. The availability of a component allowing thermal stratification would be a desirable feature for any systems thermal hydraulic code.
The simple reasoning described above and the results so obtained provided the background that leads to the reported theoretical experiment in KM.

\section{Acknowledgment}

Part of this paper is based on a conference delivered to the National Academy of Sciences at Buenos Aires, in August 23rd, 2003, on "Computational Models and Engineering Judgement (in Thermal Convection)".

\section{References}

[1] N. Aksan, "(Compiler), Best estimate methods in thermal hydraulic safety analysis," in Proceedings of the Summary and Conclusions of OECD/CSNI Seminar, Ankara, Turkey, June 1988, NEA/CSNI/R(99)22.

[2] E. Scannapieco and F. H. Harlow, "Introduction to FiniteDifference Methods for Numerical Fluid Dynamics," LA12984 (UC-700), 1995.

[3] L. M. Shotkin, "Development and assessment of U.S. nuclear regulatory commission thermal-hydraulic system computer codes," Nuclear Technology, vol. 116, no. 2, pp. 231-244, 1996.

[4] J. C. Ferreri and W. Ambrosini, "On the analysis of thermalfluid-dynamic instabilities via numerical discretization of conservation equations," Nuclear Engineering and Design, vol. 215, no. 1-2, pp. 153-170, 2002.

[5] P. Welander, "On the oscillatory instability of a differentially heated fluid loop," The Journal of Fluid Mechanics, vol. 29, part 1, pp. 17-30, 1967.

[6] "Quantifying Safety Margins: Application of Code Scaling, Applicability, and Uncertainty Evaluation Methodology to a Large Break Loss-of-Coolant Accident," NUREG/CR-5249, EGG-2659-also in Nuclear Engineering and Design, 119, 1990.

[7] F. D'Auria, "Proposal for training of thermal-hydraulic system code users," in Proceedings of the IAEA Specialist Meeting on User Qualification for and User Effect on Accident Analysis for Nuclear Power Plants, Vienna, Austria, August 1998.

[8] K. E. Carlson et al., "RELAP5/MOD3 code manual, volume I: code structure, system models and solution methods," Tech. Rep. NUREG/CR-5535, 1990.

[9] G. M. Grandi and J. C. Ferreri, "Limitations of the use of a"Heat Exchanger Approximationfor a Point Heat Source"," Internal Memo, CNEA, GSRN, Argentina, 1991.

[10] J. C. Ferreri and W. Ambrosini, "Verification of RELAP5/MOD3 with theoretical and numerical stability results on single-phase, natural circulation in a simple loop," Tech. Rep. NUREG IA/151, US Nuclear Regulatory Commission, 1999.

[11] J. C. Ferreri and A. S. Doval, "On the effeect of discretization in the computation of natural circulation in loops," Seminarios del CAMAT, vol. 24, pp. 181-212, 1984 (Spanish).

[12] P. K. Vijayan, H. Austregesilo, and V. Teschendorff, "Simulation of the unstable oscillatory behavior of single-phase natural circulation with repetitive flow reversals in a rectangular loop using the computer code athlet," Nuclear Engineering and Design, vol. 155, no. 3, pp. 623-641, 1995.

[13] W. Ambrosini and J. C. Ferreri, "Prediction of stability of one-dimensional natural circulation with a low diffusion numerical scheme," Annals of Nuclear Energy, vol. 30, no. 15, pp. 1505-1537, 2003. 
[14] W. Ambrosini, N. Forgione, J. C. Ferreri, and M. Bucci, "The effect of wall friction in single-phase natural circulation stability at the transition between laminar and turbulent flow," Annals of Nuclear Energy, vol. 31, no. 16, pp. 1833-1865, 2004.

[15] D. L. Garassa, Los Automatas y Otros Ensayos, Editorial Corregidor, Buenos Aires, Argentina, 1992.

[16] D. S. Pilkhwal, W. Ambrosini, N. Forgione, P. K. Vijayan, D. Saha, and J. C. Ferreri, "Analysis of the unstable behaviour of a single-phase natural circulation loop with one-dimensional and computational fluid-dynamic models," Annals of Nuclear Energy, vol. 34, no. 5, pp. 339-355, 2007.

[17] J. C. Ferreri and G. M. Grandi, "On expert system assisted finite-difference schemes selection in computational fluid dynamics," in Proceedings of the 6th International Conference on Numerical Methods in Laminar \& Turbulent Flows, C. Taylor, P. M. Gresho, J. Thompson, R. L. Sani, and J. Hauser, Eds., vol. 2, Pineridge Press, Swansea, UK, July 1989.

[18] R. D. King, J. Rowland, S. G. Oliver et al., "The automation of science," Science, vol. 324, no. 5923, pp. 85-89, 2009.

[19] M. Schmidt and H. Lipson, "Distilling free-form natural laws from experimental data," Science, vol. 324, no. 5923, pp. 8185, 2009.

[20] M. Schwab, M. Kerrembach, and J. Claerbout, "Making scientific computations reproducible," Computing in Science and Engineering, vol. 2, no. 6, pp. 61-67, 2000.

[21] FLUENT Inc., FLUENT 6.2 User's Guide, Centerra Resource Park, Lebanon, NH, USA, 2003. 

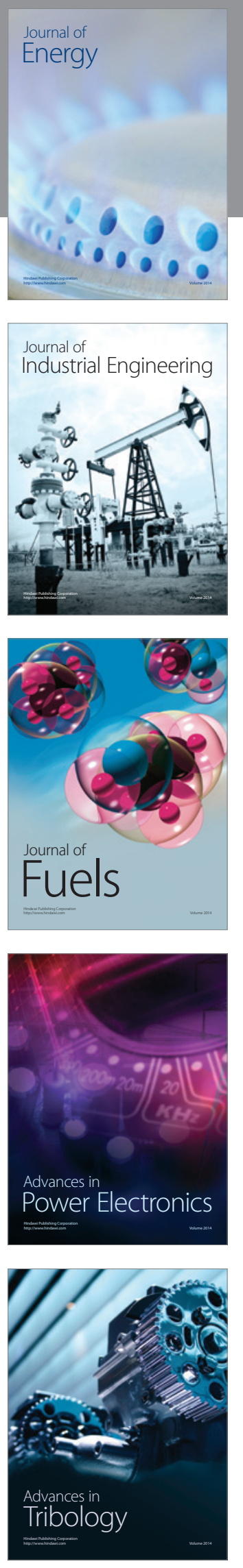
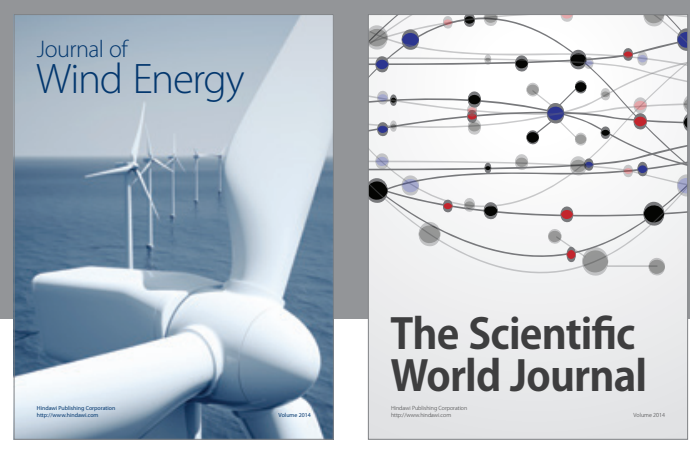

The Scientific World Journal

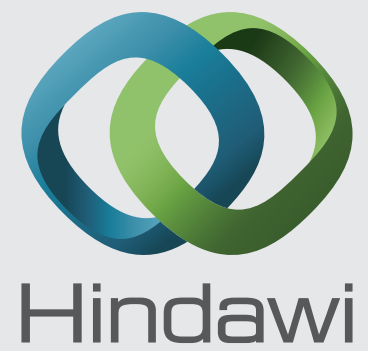

Submit your manuscripts at http://www.hindawi.com
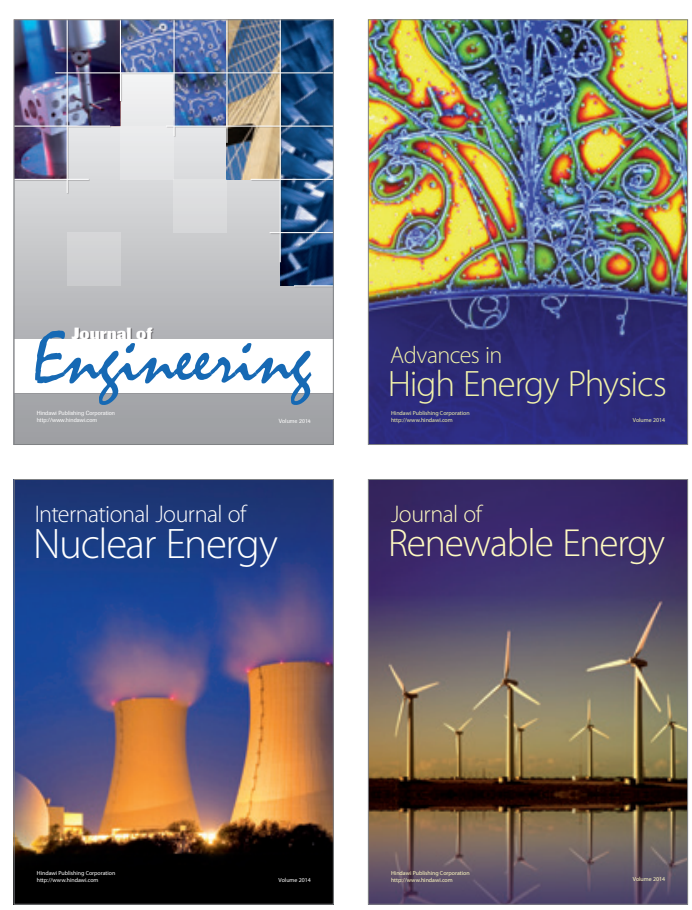

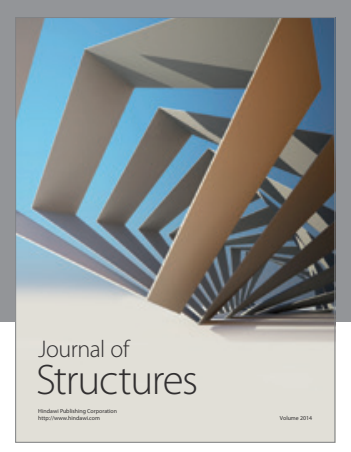

Rotating
Mechinery
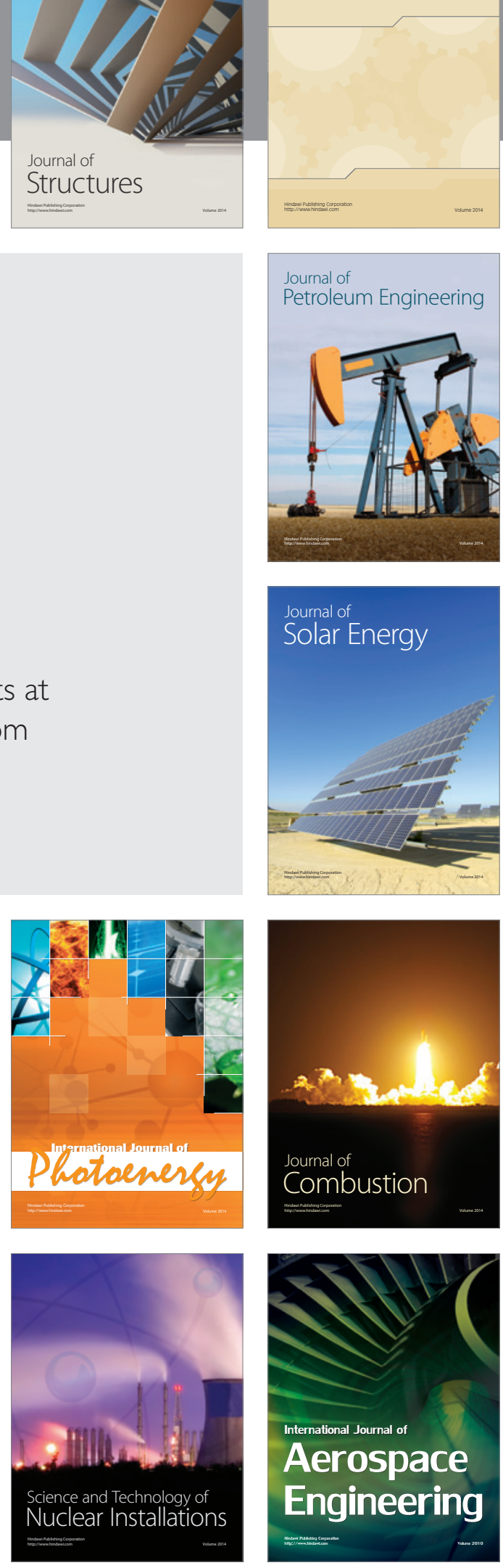